Año 11.

Revista de Investigación

Núm. 28

Académica sin Frontera

8870

ISSN: 2007 -

\title{
http://revistainvestigacionacademicasinfrontera.com
}

Recibido el 10 de octubre de 2018 Dictaminado mediante arbitraje favorablemente 17 de diciembre de 2018

\section{La operación de habilidades intelectuales con ejemplos en diferentes asignaturas del Centro Universitario Municipal de Guáimaro}

The operation of intellectual abilities with examples in different subjects of the Municipal University Center of Guáimaro.

Autor

M.Sc. Andrés Anesio Fernández-Villalobos; profesor Auxiliar. Filial Universitaria Guáimaro. Universidad de Camagüey.

Coautores

M.Sc. Fidel Brito Galindo; profesor Auxiliar. Filial Universitaria Guáimaro. Universidad de Camagüey.

M.Sc. Daitely Machado Velázquez; profesora Instructora. Filial Universitaria Guáimaro. Universidad de Camagüey.

M.Sc. Maricel Ramos García; profesora Asistente. Filial Universitaria Guáimaro. Universidad de Camagüey.

Dirección: Calle Libertad no 16 entre Iris y Unión. Guáimaro. Provincia de Camagüey. Cuba.

Código postal: 72600

Teléfono: 813489

Correo electrónico:andres.ffernandez@reduc.edu.cu

Institución: Universidad de Camagüey. 


\title{
http://revistainvestigacionacademicasinfrontera.com
}

\begin{abstract}
Resumen
El trabajo tiene como objetivo la operación de las habilidades en los objetivos de diferentes asignaturas de las carreras que se estudian en el Centro Universitario Municipal de Guáimaro, atendiendo a las dificultades comprobadas en la revisión de planes y de visitas a clases, donde se evidencia insuficiente conocimiento por parte de los docentes a la hora de utilizar la habilidad adecuada en el objetivo de una clase, y seguir el algoritmo lógico en su operacióny que conlleve a derivar las acciones inherente de la habilidad rectora, de acuerdo al contenido que tienen que aprender los estudiantes; y el mejoramiento de este aprendizaje para que sea desarrollador y significativo que produzca la obtención de un profesional altamente preparado, como el que pide la universidad contemporánea.
\end{abstract}

Palabras claves: operacionalización, habilidad, objetivo

\begin{abstract}
The work has as objective the operation of the abilities in the objectives of different subjects of the careers that are studied in the Municipal University Center of Guáimaro, assisting to the difficulties checked in the revision of plans and of visits to classes, where insufficient knowledge is evidenced on the part of the educational ones when using the ability adapted in the objective of a class, and to follow the logical algorithm in its operation and that it bears to derive the inherent actions of the ability rector, according to the content that you/they have to learn the students; and the improvement of this learning so that it is developer and significant that produces the obtaining of a highly prepared professional, as which requests the contemporary university.
\end{abstract}

Key words: Operation, ability, objective 


\section{http://revistainvestigacionacademicasinfrontera.com}

\section{Introducción}

Al hablar de las habilidades en una clase se hace imprescindible primero referirse al objetivo, ya que la habilidad forma parte intrínseca del mismo, por tanto los autores se referirán primero a los elementos contentivos del objetivo como algo esencial de los componentes no personales del proceso docente educativo y luego a su operacionalización.

Si se cumple el objetivo de una clase, entonces hay cambios significativos en el aprendizaje de los estudiantes; si los docentes saben redactar el objetivo y llevar correctamente las operaciones con las habilidades la clase será fructífera.

En el Centro Universitario Municipal (CUM) de Guáimaro, existe un número creciente de profesores a tiempo parcial, algunos de ellos no provienen de carreras pedagógicas, con problemas didácticos y otros que son licenciados en educación.

\section{Materiales y métodos}

Se utilizaron métodos empíricos como la observación de planeamientos y clases de los docentes; además del intercambio con los mismos que permitió constatar los conocimientos que poseen respecto a la redacción de los objetivos y la selección correcta de las habilidades intelectuales.

Histórico-lógico, permitió caracterizar las etapas del desarrollo histórico de las habilidades y competencias en el proceso de formación del profesional de la docencia en Cuba.

El hipotético-deductivo para la formulación de la idea a defender.

La población estuvo conformada por 18 docentes a tiempo completo y parcial del CUM Guáimaro. 


\section{http://revistainvestigacionacademicasinfrontera.com}

\section{Resultados}

En revisiones de planeamientos y visitas a clases efectuadas por los coordinadores y jefes de carreras, así como por el director y subdirector del centro se han detectado las siguientes insuficiencias:

- Limitado conocimiento por parte de los docentes a la hora de utilizar la habilidad adecuada en el objetivo de una clase.

- Insuficiente conocimiento en seguir el algoritmo lógico en las operaciones con las habilidades.

- Dificultades en derivar las acciones inherentes de la habilidad rectora.

Esto justifica el artículo que se expone.

\section{Planteamiento del problema}

Como objeto de esta investigación se tiene el proceso docente educativo de la enseñanza universitaria de los cursos por encuentro para trabajadores; como campo la operación de las habilidades intelectuales de las asignaturas vigentes en las carreras del Centro Universitario Municipal de Guáimaro, idea a defender, la operacionalización de habilidades intelectuales en diferentes asignaturas es condición indispensable para el desarrollo exitoso de la labor de los docentes; teniendo entonces como objetivo, ejemplificar la operación de las habilidades intelectuales con muestras en diferentes asignaturas del Centro Universitario Municipal de Guáimaro.

Para llevar a cabo esta investigación se han ido realizando paulatinamente una serie de tareas investigativas entre las que se encuentran:

1. Caracterización del estado actual de las habilidades intelectuales en las diferentes asignaturas.

2. Estudio de los fundamentos teóricos generales que sirven de base para la operacionalización de las habilidades intelectuales. 
Año 11.

Revista de Investigación

Núm. 28

Académica sin Frontera

8870

ISSN: 2007 -

\section{http://revistainvestigacionacademicasinfrontera.com}

4. Elaboración de los ejemplos de operacionalización de las habilidades en las diferentes asignaturas.

5. Valoración del impacto de la ejemplificación de operacionalización de habilidades intelectuales en el proceso docente educativo.

\section{Discusión}

El desarrollo a alcanzar en las habilidades intelectuales de los estudiantes en su carrera, que les permita actuar con independencia y creatividad en su futura actividad profesional, presume el conocimiento adecuado de los docentes para orientar la operacionalización de las habilidades acorde al tipo de curso para trabajadores de nuestro centro.

Podemos preguntarnos ¿qué es el objetivo de una clase?

El Dr. Alejandro Barba Carrazco dice: "Si no tienes claro adónde vas, lo más probable es que acabes en otro sitio.", por tanto el objetivo es el resultado a alcanzar, la meta propuesta, debe estar correctamente redactado, sin ambages; tiene un carácter recto.

Según Madeleine SalellasBrínguez, el objetivo es lo que se quiere lograr en el estudiante, son los propósitos y aspiraciones que se pretende formar en los alumnos. Debe redactarse en términos de aprendizaje, es decir, que tanto para el docente, como para el alumno, el objetivo es el mismo y está en función de este último.

El objetivo es el componente rector del proceso docente-educativo, o lo que es lo mismo, el más importante, porque es él, el que en un lenguaje pedagógico, explícita la solución de los problemas, de la necesidad social, el objetivo es el modelo pedagógico del encargo social

Carlos Álvarez de Zayas, muy sabiamente plantea: "El objetivo es el modelo pedagógico del encargo social, es la aspiración, el propósito que la institución docente se propone alcanzar en los estudiantes."

Los objetivos se proyectan de acuerdo con el grado de trascendencia en la transformación que se espera alcanzar en los estudiantes en estas tres dimensiones; en el objetivo se 
Año 11.

Revista de Investigación

Núm. 28

Académica sin Frontera

8870

ISSN: 2007-

\section{http://revistainvestigacionacademicasinfrontera.com}

concretan estas dimensiones en las siguientes funciones: instructiva, desarrolladora y educativa.

INSTRUCTIVA: $($ qué) = Asimilación por el estudiante de un conocimiento y por tanto del dominio de una habilidad.

DESARROLLADORA: $($ cómo) $=$ Transformaciones que en las potencialidades del modo de actuación se quiere alcanzar en los alumnos.

EDUCATIVA: (para qué) = Transformaciones a lograr en los sentimientos, las convicciones y otros rasgos de la personalidad de los escolares.

El objetivo expresa en su estructura el qué, el cómo y el para qué, lo que se explica a continuación:

QUÉ: conocimiento y habilidad ( esta última precisa del conocimiento por parte del docente de su clasificación, tipología y operacionalización)

CÓMO: modo de actuación

PARA QUÉ: finalidad (educación ideo-política, ciudadana, vocacional y profesional, y científica.

Los elementos de un objetivo correctamente estructurado se manifiestan en el siguiente ejemplo:

[Instruir] a los profesionales [formación pedagógica y didáctica], [a través de talleres] [para un mejor resultado en el proceso enseñanza-aprendizaje.]

Donde:

A. [Instruir]: es la habilidad

B. [formación pedagógica y didáctica]: es el qué

C. [a través de talleres]: es el cómo

D. [para un mejor resultado en el proceso enseñanza-aprendizaje]: es el para qué

Hay diferentes acepciones de habilidad, por ejemplo en un trabajo publicado por Carlos Manuel Cañedo y Maritza Cáceres Mesa plantean que: 
Año 11.

Revista de Investigación

Núm. 28

Académica sin Frontera

8870

ISSN: 2007-

\section{http://revistainvestigacionacademicasinfrontera.com}

En la literatura pedagógica y psicológica, el término habilidades aparece con diferentes acepciones:

- Es el sistema de acciones y operaciones dominado por el sujeto que responde a un objetivo

- Es la capacidad adquirida por el hombre, de utilizar creadoramente sus conocimientos y hábitos tanto en el proceso de actividad teórica como práctica

- Significa el domino de un sistema complejo de actividades psíquicas, lógicas y prácticas, necesarias para la regulación conveniente de la actividad, de los conocimientos y hábitos que posee el sujeto

- Es la asimilación por el sujeto de los modos de realización de la actividad, que tienen como base un conjunto determinado de conocimientos y hábitos.

Las habilidades son el grado de competencia de un sujeto concreto frente a un objetivo determinado. Es decir, en el momento en el que se alcanza el objetivo propuesto en la habilidad.

En cada habilidad se pueden determinar las operaciones cuya integración permite el dominio por los estudiantes de un modo de actuación. Una misma acción puede formar parte de distintas habilidades, así como una misma habilidad puede realizarse a través de diferentes acciones. Las acciones se correlacionan con los objetivos, mientras que las operaciones lo hacen con las condiciones.

Pero ¿qué es una habilidad intelectual?

Según C. Pozo Coll y otros autores, "Un conjunto de acciones ordenadas, orientadas a la consecución de una meta" observándose aquí las siguientes características:

- Que se refieren a una actuación.

- Que es una actuación ordenada.

- Que esta actuación se orienta hacia la consecución de una meta”. 
Año 11.

Revista de Investigación

Núm. 28

Académica sin Frontera

8870

ISSN: 2007-

\section{http://revistainvestigacionacademicasinfrontera.com}

Ladislao Romero Bojórquez plantea que las habilidades intelectuales son: "Un conjunto de aptitudes que optimizan el aprendizaje de nuevos conocimientos; complementando estas habilidades con habilidades manuales, estéticas, y demás propias del ser humano, que mostrando una actitud humanista favorecen en gran medida en el proceso de aprendizaje de nuevas habilidades en el ser humano".

Por tanto nos referiremos a las habilidades intelectuales más comunes en el proceso docente educativo:

En ellas observaremos las acciones necesarias realizar a partir de su composición, otras habilidades que son necesario accionar para alcanzar la habilidad principal propuesta en el objetivo de las clases:

\section{SINTETIZAR}
a) Estudiar cada parte determinada.
b) Determinar los límites del objeto a analizar (todo).
c) Determinar los criterios de descomposición del todo.
d) Determinar las partes del todo.

\section{ANALIZAR}
a) Comparar las partes entre sí (rasgos comunes y diferencias).
b) Describir los nexos entre las partes (causales, de condicionalidad, etc.).
c) Elaborar conclusiones acerca de la integralidad del todo.

\section{COMPARAR}

a) Determinar los objetos de comparación.

b) Determinar las líneas o parámetros de comparación.

c) Determinar las diferencias y las semejanzas entre los objetos, entre cada línea de comparación.

d) Elaborar conclusiones acerca de cada objeto de comparación y líneas (síntesis parcial). 
Año 11.

Revista de Investigación

Núm. 28

Académica sin Frontera

8870

ISSN: 2007-

e) Elaborar conclusiones generales.

\section{DETERMINAR LO ESENCIAL}

a) Analizar el objeto de estudio.

b) Comparar entre sí las partes del todo.

c) Descubrir lo determinante, lo fundamental, lo estable del todo.

d) Revelar los nexos entre los rasgos esenciales.

\section{ABSTRAER}

a) Analizar el objeto de abstracción.

b) Determinar lo esencial.

c) Despreciar los rasgos y nexos secundarios, no determinantes del objeto.

\section{CARACTERIZAR}
a) Analizar el objeto.
b) Determinar lo esencial del objeto.
c) Comparar con otros objetos de su clase y de otras clases.
d) Seleccionar los elementos que lo tipifican y distinguen de los demás objetos.

\section{DEFINIR}

a) Determinar las características esenciales que distinguen y determinan el objeto de definición.

b) Enumerar de forma sintética y precisa los rasgos esenciales del objeto.

\section{IDENTIFICAR}
a) Analizar el objeto.
b) Caracterizar el objeto.
c) Establecer la relación del objeto con un hecho, concepto o ley de los conocidos.

\section{CLASIFICAR}
a) Identificar el objeto de estudio.
b) Seleccionar los criterios o fundamentos de clasificación.
c) Agrupar los elementos en diferentes clases o tipos. 
Año 11.

Revista de Investigación

Núm. 28

Académica sin Frontera

8870

10. ORDENAR

http://revistainvestigacionacademicasinfrontera.com

a) Identificar el objeto de estudio.

b) Seleccionar el o los criterios de ordenamiento(lógico, cronológico, etc.)

c) Clasificar los elementos según el criterio de ordenamiento.

d) Ordenar los elementos

11. ARGUMENTAR
a) Interpretar el juicio de partida.
b) Encontrar de otras fuentes los juicios que corroboran el juicio inicial.
c) Seleccionar las reglas lógicas que sirven de base al razonamiento.

\section{GENERALIZAR}
a) Determinar lo esencial en cada elemento del grupo a generalizar
b) Comparar los elementos.
c) Seleccionar los rasgos, propiedades o nexos esenciales y comunes a todos los elementos.
d) Clasificar y ordenar estos rasgos.
e) Definir los rasgos generales del grupo.

\section{OBSERVAR}
a) Determinar el objeto de observación.
b) Determinar los objetivos de la observación.
c) Fijar los rasgos y características del objeto observado con relación a los objetivos.

\section{DESCRIBIR}
a) Determinar el objeto a describir.
b) Observar el objeto.
c) Elaborar el plan de descripción(ordenamiento lógico de los elementos a describir).
d) Reproducir las características del objeto siguiendo el plan.

\section{RELATAR O NARRAR}

a) Delimitar el período temporal de acontecimientos a relatar.

b) Seleccionar el argumento del relato(acciones que acontecen como hilo conductor de la narración en el tiempo). 
Año 11.

Revista de Investigación

Núm. 28

Académica sin Frontera

8870

ISSN: 2007 -

\section{http://revistainvestigacionacademicasinfrontera.com}

c) Caracterizar los demás elementos que dan vida y condiciones concretas al argumento(personajes históricos, relaciones espacio temporales,etc.).

d) Exponer ordenadamente el argumento y el contenido.

16. ILUSTRAR

a) Determinar el concepto, regularidad o ley que se quiere ilustrar.

b) Seleccionar los elementos factuales (a partir de criterios lógicos y de la observación, descripción, relato y otras fuentes).

c) Establecer las relaciones de correspondencia de lo factual con lo lógico.

d) Exponer ordenadamente las relaciones encontradas.

\section{VALORAR}
a) Caracterizar el objeto de valoración.
b) Establecer los criterios de valoración (valores).
c) Comparar el objeto con los criterios de valor establecidos.
d) Elaborar los juicios de valor acerca del objeto.

\section{CRITICAR}
a) Caracterizar el objeto a criticar.
b) Valorar el objeto a criticar.
c) Argumentar los juicios de valor elaborados.
d) Refutar las tesis de partida del objeto de crítica con los argumentos.

\section{RELACIONAR}
a) Analizar de manera independiente los objetos a relacionar.
b) Determinar los criterios de relación entre los objetos.
c) Determinar los nexos de un objeto hacia otro a partir de los criterios seleccionados (elaborar síntesis parcial).
d) Determinar los nexos inversos (elaborar síntesis parcial).
e) Elaborar las conclusiones generales.

\section{RAZONAR}

a) Determinar las premisas (juicios o criterios de partida). 
Año 11.

Revista de Investigación

Núm. 28

Académica sin Frontera

8870

ISSN: 2007 -

\section{http://revistainvestigacionacademicasinfrontera.com}

b) Encontrar la relación de inferencia entre las premisas a través del término medio.

c) Elaborar las conclusiones (nuevo juicio obtenido.

\section{INTERPRETAR}

a) Analizar el objeto o información.

b) Relacionar las partes del objeto.

c) Obtener la lógica de las relaciones encontradas.

d) Elaborar las conclusiones acerca de los elementos, relaciones y razonamientos que aparecen en el objeto o información a interpretar.

\section{EXPLICAR}

a) Interpretar el objeto o información.

b) Argumentar los juicios de partida.

c) Establecer las interrelacionesde los argumentos.

\section{DEMOSTRAR}

a) Caracterizar el objeto de demostración.

b) Seleccionar los argumentos y hechos que corroboran el objeto de demostración.

c) Elaborar los razonamientos que relacionan los argumentos que muestran la veracidad del objeto de demostración.

Pero ¿qué entonces operacionalizar?

La operacionalización es un proceso que consiste en dar salida a un grupo de acciones (habilidades secundarias) para dar cumplimiento al objetivo de una clase desde la habilidad rectora.

Otra forma de representar un grupo de habilidades intelectuales es mediante este cuadro donde se clasifican en descriptivas, analíticas, críticas y creativas. 
Año 11.

Revista de Investigación

Núm. 28

Académica sin Frontera

8870

ISSN: 2007 -

$\underline{\text { http://revistainvestigacionacademicasinfrontera.com }}$

\begin{tabular}{|c|c|c|c|}
\hline \multicolumn{5}{|c|}{ HABILIDADES INTELECTUALES } \\
\hline DESCRIPTIVAS & ANALITICAS & CRÍTICAS & CREATIVAS \\
\hline Contar & Clasificar & Evaluar & Transformar \\
\hline Resumir & Relacionar & Enjuiciar & Aplicar \\
\hline Enumerar & Analizar & Criticar & Imaginar \\
\hline Resaltar & Agrupar & Elegir & Inventar \\
\hline Describir & Comparar & Apreciar & Diseñar \\
\hline Narrar & Generalizar & Justificar & Elaborar \\
\hline
\end{tabular}

A partir de lo anteriormente expuesto los autores presentan los ejemplos de la operación de habilidades en diferentes asignaturas que se imparten en nuestro centro.

Ojalá sea de provecho para quien estudie y analice este artículo. 


\section{Ejemplos de la operación con las habilidades en los objetivos}

\section{(Jullo- viciempre LU18)}

\begin{tabular}{|c|c|c|c|c|c|}
\hline 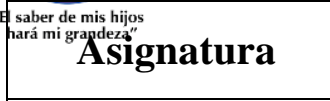 & Carrera & Contenido & Objetivo & Habilidad & $\begin{array}{l}\text { Operacionalización de } \\
\text { Revlstłłdslididectigación }\end{array}$ \\
\hline $\begin{array}{l}\text { Año 11. } \\
\text { Núm. } 28\end{array}$ & $\begin{array}{l}\text { Cultura } \\
\text { Física }\end{array}$ & $\begin{array}{l}\text { Medidas de tendencia } \\
\text { Central o estadígrafos } \\
\text { de posiciónttipddrievistai } \\
\text { aritmética, mediana, } \\
\text { moda, cuartiles y } \\
\text { percentiles. } \\
\text { Estadígrafos de } \\
\text { dispersión: recorrido, } \\
\text { varianza, desviación } \\
\text { típica, mínimo, } \\
\text { máximo, coeficiente de } \\
\text { variación y regla de las } \\
\text { tres sigmas. }\end{array}$ & 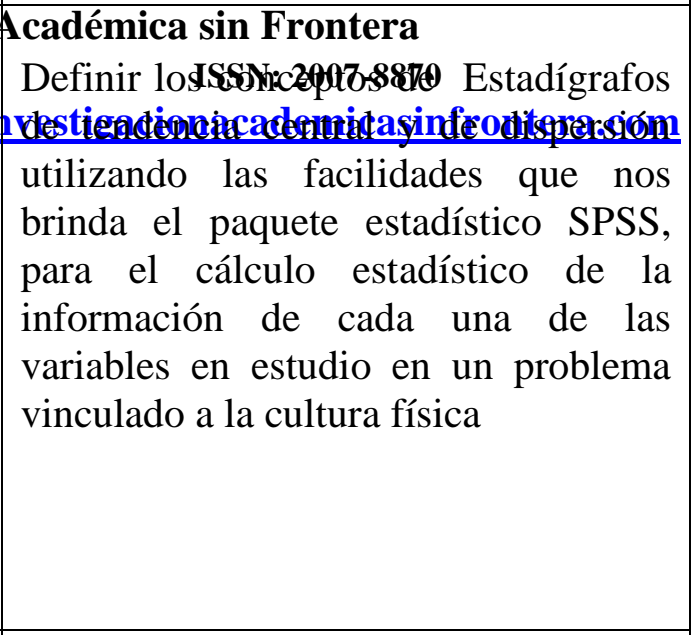 & 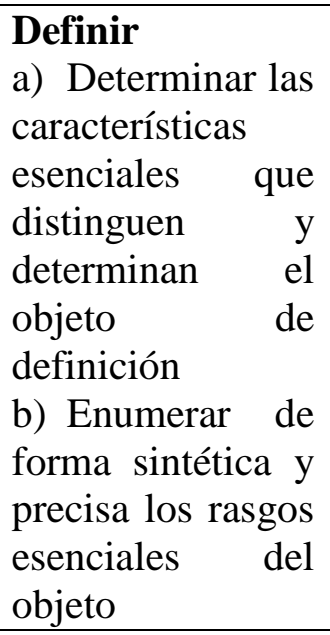 & $\begin{array}{l}\text { a) Determinación las } \\
\text { características que } \\
\text { distinguen a los } \\
\text { estadígrafos de tendencia } \\
\text { central y de dispersión. } \\
\text { b) Enumeración de los } \\
\text { rasgos que tipifican cada } \\
\text { estadígrafo. }\end{array}$ \\
\hline $\begin{array}{l}\text { Matemática } \\
\text { Financiera }\end{array}$ & Contabilidad & $\begin{array}{l}\text { Cálculo de magnitudes } \\
\text { derivadas del interés } \\
\text { compuesto a través de } \\
\text { diferentes métodos. }\end{array}$ & $\begin{array}{l}\text { Calcular las magnitudes derivadas del } \\
\text { Interés Compuesto a través de tablas de } \\
\text { diferentes períodos y disímiles tasas } \\
\text { para el aprendizaje de diferentes } \\
\text { métodos. }\end{array}$ & $\begin{array}{l}\text { Calcular } \\
\text { a) Identificar los } \\
\text { objetos del } \\
\text { cálculo. } \\
\text { b) Caracterizar } \\
\text { de los objetos del } \\
\text { cálculo. } \\
\text { c) Determinar la } \\
\text { operación u } \\
\text { operaciones que } \\
\text { se establecen } \\
\text { entre los objetos } \\
\text { del cálculo. }\end{array}$ & $\begin{array}{l}\text { a) Identificación las } \\
\text { magnitudes. } \\
\text { b) Caracterización de las } \\
\text { magnitudes derivadas del } \\
\text { interés compuesto. } \\
\text { c) Determinación de las } \\
\text { operaciones a realizar. } \\
\text { d) Caracterización de las } \\
\text { operaciones que se } \\
\text { establecen entre las } \\
\text { magnitudes. } \\
\text { e) Caracterización de las } \\
\text { operaciones que se }\end{array}$ \\
\hline
\end{tabular}




\section{ISSN: 2007-8870}

Revista de Investigación

Núm. 28

http://revistainvestigacionacademicasinfrontera.com

\begin{tabular}{|c|c|c|c|c|c|}
\hline & & & & $\begin{array}{l}\text { d) Caracterizar } \\
\text { las operaciones } \\
\text { que se establecen } \\
\text { entre los objetos } \\
\text { del cálculo. } \\
\text { e) Determinación } \\
\text { de los nexos que } \\
\text { se establecen } \\
\text { entre las } \\
\text { operaciones. } \\
\text { f) Establecer el } \\
\text { algoritmo que } \\
\text { representa a la } \\
\text { operación } \\
\text { operaciones. } \\
\text { g) Ejecutar el } \\
\text { algoritmo. } \\
\text { h) Comprobar la } \\
\text { resultatividad del } \\
\text { algoritmo. }\end{array}$ & $\begin{array}{l}\text { establecen entre las } \\
\text { magnitudes. } \\
\text { f) Establecimiento del } \\
\text { algoritmo que representa } \\
\text { los cálculos a realizar. } \\
\text { g) Ejecución del } \\
\text { algoritmo } \\
\text { h) Comprobación de los } \\
\text { resultados obtenidos. }\end{array}$ \\
\hline Pedagogía & Primaria & $\begin{array}{l}\text { Rol profesional del } \\
\text { maestro: Definición. } \\
\text { Sus tareas básicas y } \\
\text { funciones en el proceso } \\
\text { educativo de niños } \\
\text { adolescentes y jóvenes. }\end{array}$ & $\begin{array}{l}\text { Caracterizar el rol profesional del } \\
\text { maestro a través de su misión en la } \\
\text { escuela, sus tareas básicas y funciones } \\
\text { con el fin de elevar su desempeño } \\
\text { profesional pedagógico. }\end{array}$ & $\begin{array}{l}\text { Caracterizar } \\
\text { a) Analizar el } \\
\text { objeto } \\
\text { b) Determinar } \\
\text { lo esencial del } \\
\text { objeto } \\
\text { c) Comparar } \\
\text { con otros objetos }\end{array}$ & $\begin{array}{l}\text { a) Análisis los } \\
\text { conceptos de modelos del } \\
\text { profesional, rol } \\
\text { profesional, modos de } \\
\text { actuación profesionales } \\
\text { consonancia con la ética } \\
\text { profesional pedagógica. }\end{array}$ \\
\hline
\end{tabular}


Año 11.

Núm. 28

http://revistainvestigacionacademicasinfrontera.com

\begin{tabular}{|c|c|c|c|c|c|}
\hline & & & & $\begin{array}{l}\text { de su clase y de } \\
\text { otras clases } \\
\text { d) Seleccionar } \\
\text { los elementos que } \\
\text { lo tipifican y } \\
\text { distinguen de los } \\
\text { demás objetos }\end{array}$ & $\begin{array}{l}\text { b) Determinación las } \\
\text { tareas y funciones el } \\
\text { maestro. } \\
\text { c) Comparar con } \\
\text { docentes de otros niveles. } \\
\text { d) Selección los rasgos } \\
\text { esenciales del maestro } \\
\text { primario. }\end{array}$ \\
\hline Pedagogía & Primaria & $\begin{array}{l}\text { El objeto de estudio de } \\
\text { la Pedagogía. } \\
\text { Categorías } \\
\text { pedagógicas.Leyes y } \\
\text { principios. }\end{array}$ & $\begin{array}{l}\text { Argumentar el sistema categorial de la } \\
\text { Pedagogía como ciencia, a partir de su } \\
\text { objeto de estudio, leyes, métodos y } \\
\text { categorías, evidenciando sus relaciones } \\
\text { sistémicas y su significación para su } \\
\text { futuro desempeño profesional. }\end{array}$ & \begin{tabular}{|l}
\multicolumn{2}{|c}{ Argumentar } \\
a) Interpretar el \\
juicio de partida. \\
b) Encontrar de \\
otras fuentes los \\
juicios $\quad$ que \\
corroboran el \\
juicio inicial. \\
c) Seleccionar \\
las reglas lógicas \\
que sirven de base \\
al razonamiento.
\end{tabular} & $\begin{array}{l}\text { a) Interpretación del } \\
\text { objeto de estudio de la } \\
\text { Pedagogía. } \\
\text { b) Búsqueda } \\
\text { diferentes fuentes sobre } \\
\text { el tema. } \\
\text { c) Establecimiento de } \\
\text { relaciones entre las } \\
\text { categorías pedagógicas. } \\
\text { d) Emisión de juicios } \\
\text { acerca de la importancia } \\
\text { de este conocimiento } \\
\text { para su futuro desempeño } \\
\text { profesional. }\end{array}$ \\
\hline $\begin{array}{l}\text { Anatomía, } \\
\text { Fisiología e } \\
\text { higiene del } \\
\text { desarrollo }\end{array}$ & Preescolar & $\begin{array}{lc}\text { Tratamiento a los } \\
\text { sistemas } & \text { nerviosos, } \\
\text { sensoriales y } & \text { motores, }\end{array}$ & $\begin{array}{l}\text { Caracterizar los sistemas sensoriales en } \\
\text { la primera infancia a través de la } \\
\text { presentación electrónica para el }\end{array}$ & $\begin{array}{l}\text { Caracterizar } \\
\text { a) Analizar el } \\
\text { objeto }\end{array}$ & $\begin{array}{l}\text { a) Análisis r los } \\
\text { conceptos de sistemas } \\
\text { nerviosos, sensoriales y } \\
\text { motores. }\end{array}$ \\
\hline
\end{tabular}

\section{Académica sin Frontera}

ISSN: 2007-8870

Revista de Investigación

Revista de Investigación 
Año 11.

Núm. 28
Académica sin Frontera

ISSN: 2007-8870

http://revistainvestigacionacademicasinfrontera.com

\begin{tabular}{|c|c|c|c|c|c|}
\hline & & $\begin{array}{l}\text { así como los trastornos } \\
\text { más frecuentes. }\end{array}$ & $\begin{array}{l}\text { perfeccionamiento de su labor como } \\
\text { profesional de la educación preescolar. }\end{array}$ & $\begin{array}{l}\text { b) Determinar lo } \\
\text { esencial del } \\
\text { objeto } \\
\text { c) Comparar con } \\
\text { otros objetos de } \\
\text { su clase y de } \\
\text { otras clases } \\
\text { d) Seleccionar } \\
\text { los elementos que } \\
\text { lo tipifican y } \\
\text { distinguen de los } \\
\text { demás objetos }\end{array}$ & $\begin{array}{l}\text { b) Determinación de } \\
\text { las funciones de cada } \\
\text { sistema. } \\
\text { c) Comparaciónestos } \\
\text { sistemas. } \\
\text { d) Selección de los } \\
\text { rasgos esenciales de cada } \\
\text { sistema. }\end{array}$ \\
\hline
\end{tabular}

\section{Revista de Investigación}

Revista de Investigación 
Año 11.

Académica sin Frontera

Núm. 28

ISSN: 2007-8870

\section{http://revistainvestigacionacademicasinfrontera.com}

\section{CONLUSIONES}

- El diagnóstico del estado actual corroboró que los profesores y estudiantes desconocen con precisión las habilidades intelectuales y su operacionalización.

- Fue elaborado un grupo de ejemplos donde desde el contenido, el objetivo y la habilidad rectora se demostró la posibilidad de su operacionalización adecuada.

- Los docentes mostraron satisfacción con los ejemplos que coadyuvaron al mejoramiento del proceso docente educativo.

- Se evidenció el cambio en el modo de actuación de los docentes en las nuevas visitas realizadas a las clases, además de la revisión exhaustiva de los planes de clases.

- En visitas realizadas a los colectivos de asignaturas en el centro se evidenció el trabajo sistemático llevado a cabo por los profesores que viabiliza la preparación de la clase encuentro redundando clases científicamente articuladas que posibilitan el mejoramiento del proceso docente educativo.

\section{BIBLIOGRAFÍA}

1. Argudín, Yolanda.(1997) Las habilidades en la educación. En: Didáctica.29, Méjico,--p.1

2. Coll, C. Pozo, I Sarabia, J. y Valls, D. (1992). Los contenidos en la Reforma. España: Santillana.

3. Crispin, María Luisa. (1997). Sobre el desarrollo de habilidades en el currículum para lograr una educación integral./ María Luisa Crispín, Isabel Cruz.--En Didáctica. -- No. 29.-- México D.F., -p.52

4. Elementos de Matemática Financiera. Juan Carlos Domínguez Tomo I

5. García Muñoz, José J. (2004). Modelo teórico - metodológico para el perfeccionamiento del proceso de enseñanza - aprendizaje del cálculo aritmético en el primer ciclo de la enseñanza primaria. Tesis en opción al título de doctor de Ciencias Pedagógicas. Pág. 7.

6. Martínez Rubio, Blanca N y otros. El trabajo metodológico en la estrategia de formación inicial y permanente de los profesionales de la educación. Curso 66. Sello Editor Educación Cubana. La Habana. p. 37.

7. Ministerio de educación: Formación y desarrollo de capacidades y habilidades. (1989.). En 
Año 11.

\section{Académica sin Frontera}

Revista de Investigación

Núm. 28

ISSN: 2007-8870

\section{http://revistainvestigacionacademicasinfrontera.com}

Seminario Nacional a Dirigentes, Metodólogos e Inspectores de las Direcciones Provinciales y Municipales de Educación y de los Institutos Superiores Pedagógicos. Febrero, Ciudad de la Habana. (Documentos normativos y metodológicos).

8. Petrovski, a.: Psicología General. Manual didáctico para los Institutos de Pedagogía. Ed. Progreso. Moscú, 1976.

9. Plasencia Moro, ZanettiLecuona y García Álvarez (1989): Metodología de la Investigación Histórica. p.48.

10. Ruiz Aguilera Ariel, es uno de los investigadores que los propone a partir de que estos procesos lógicos del pensamiento están en todos los demás métodos de investigación.

11. SalellasBrínguez, Madeleine. Algunas consideraciones acerca del objetivo de enseñanza como categoría pedagógica del proceso de enseñanza-aprendizaje. En: Revista Transformaciones. Vol II. No.18. julio-diciembre de 2009.ISSN: 2077-2890

\section{Como citar este articulo}

Andrés Anesio Fernández-Villalobos, Fidel Brito Galindo, Daitely Machado Velázquez, Maricel Ramos García. La operación de habilidades intelectuales con ejemplos en diferentes asignaturas del Centro Universitario Municipal de Guáimaro. RIASF. Núm. 28, Julio-diciembre (2018), ISSN 2007-8870. pp. xx-xx. 